\title{
O ENFERMEIRO - LÍDER NO GERENCIAMENTO DE RISCO PARA PREVENÇÃO E CONTROLE DE INFECÇÕES EM PACIENTES COM CÂNCER*
}

\author{
Nádia Fontoura Sanhudo ${ }^{1}$, Marléa Chagas Moreira ${ }^{2}$
}

\begin{abstract}
RESUMO: O estudo objetivou elaborar estratégias de liderança em enfermagem voltadas à incorporação de medidas de prevenção e controle de infecções relacionadas à assistência à saúde em pacientes com câncer. Pesquisa Convergente Assistencial desenvolvida num processo interativo com nove enfermeiros, em 2013, num hospital oncológico do interior de Minas Gerais, com tratamento dos dados por meio de software. Emergiram cinco classes que representam as tendências das estratégias. A classe 1: a presença do enfermeiro-líder nos setores de internação; classe 2: o processo de trabalho do enfermeiro-líder; classe 3: a higienização das mãos; classe 4: o enfermeiro da comissão de controle de infecção hospitalar como parceiro do enfermeiro-líder; e classe 5: a promoção do conhecimento sobre infecções para a equipe de enfermagem. Para os enfermeiros a elaboração das estratégias de liderança é aposta empreendida de acordo com a situação, para dar conta da imprevisibilidade presente no contexto e a necessidade de adotar atitude educativa.

DESCRITORES: Enfermagem oncológica; Liderança; Infecção hospitalar; Gerenciamento de segurança; Segurança do paciente.

\section{THE NURSE-LEADER IN THE MANAGEMENT OF RISK FOR THE PREVENTION AND CONTROL OF INFECTIONS IN PATIENTS WITH CANCER}

ABSTRACT: This study aimed to develop nursing leadership strategies directed towards the incorporation of measures for the prevention and control of healthcare-associated infectionsin patients with cancer. It involvedConvergent Care Researchundertaken in an interactive process with nine nurses, in 2013, in an oncological hospital in the interior of Minas Gerais, with treatment of the data through software. Five classes emerged, representing the tendencies of the strategies. Class 1: the presence of the nurse-leader in the inpatient departments; class 2: the work process of the nurse-leader; class 3: handwashing; class 4: the nurse of the hospital infection control committee as a partner of the nurse-leader; and class 5: the promotion of knowledge regarding infections for the nursing team. For the nurses, the elaboration of the leadership strategies is a bet used according to the situation, in order to take account of the unpredictability present in the context and the need to adopt an educational attitude.

DESCRIPTORS: Oncology nursing; Leadership; Cross infection; Safety management; Patient safety.
\end{abstract}

\section{EL ENFERMERO - LÍDER EN LAGESTIÓN DE RIESGO PARA PREVENCIÓNY CONTROL DE INFECCIONES EN PACIENTES CON CÁNCER}

RESUMEN: Estudo cuyo objetivo fue elaborar estrategias de liderazgo en enfermería referentes a la adopción de medidas de preveción y control de infecciones asociadas a la asistencia a la salud en pacientes con cáncer. Investigación Convergente Asistencial desarrollada por proceso interactivo con nueve enfermeros, en 2013, en hospital oncológico del interior de Minas Gerais. El tratamiento de los datos fue hecho por medio de software. Resultaron del estudio cinco clases que representan las tendencias de las estrategias. Clase1: presencia del enfermero-líder en los sectores de internación; clase 2: proceso de trabajo del enfermerolíder; clase 3: higienización de las manos; clase 4: enfermero de la comisión de control de infección hospitalar como asociado del enfermero-líder; y clase 5: promoción del conocimiento acerca de infecciones para el equipo de enfermería. Para los enfermeros, la elaboración de estrategias de liderazgo es una apuesta hecha de acuerdo con la situación, para abarcar la imprevisibilidad presente en el contexto y la necesidad de adoptar actitude educativa.

DESCRIPTORES: Enfermería oncológica; Liderazgo; Infección hospitalar; Administración de seguridad; Seguridad del paciente. *Artigo extraído da tese de doutorado intitulada: "Estratégias de liderança em enfermagem na prevenção e controle de
infecções em pacientes com câncer". Universidade Federal do Rio de Janeiro, 2013.

${ }^{1}$ Enfermeira. Doutora em Enfermagem. Docente da Faculdade de Enfermagem da Universidade Federal de Juiz de Fora. Juiz de Fora/MG, Brasil.

${ }^{2}$ Enfermeira. Doutora em Enfermagem. Docente da Escola de Enfermagem Anna Nery da Universidade Federal do Rio de Janeiro. Rio de Janeiro, RJ, Brasil.

Autor Correspondente:

Nádia Fontoura Sanhudo

Universidade Federal de Juiz de Fora

R. José Lourenço Kelmer, s/n - 36036-900 - Juiz de Fora, MG, Brasil

E-mail: nadiasanhudo@gmail.com
Recebido: 01/03/2016

Finalizado: $14 / 09 / 2016$ 


\section{INTRODUÇÃO}

No Brasil, um marco importante para garantir a segurança do paciente foi a publicação do Programa Nacional de Segurança do Paciente (PNSP), cujo objetivo é prevenir e reduzir a incidência de eventos adversos relacionados à assistência nos serviços de saúde ${ }^{(1)}$. Entre os eventos adversos citados no PNSP, destaca-se a infecção relacionada à assistência à saúde (IRAS) categorizada como evitável, ou seja, cujo risco pode ser gerenciado.

Entende-se como relevante abordar a temática prevenção e controle de IRAS por ser uma questão persistente e de interesse para todos os cenários de prestação de assistência à saúde. Na atenção oncológica, é prioridade nas agendas de pesquisa e na prática assistencial, diante do risco das infecções que repercutem em altas taxas de incidência e mortalidade nessa clientela ${ }^{(2-3)}$.

O termo risco refere-se à chance de exposição dos pacientes com câncer à ocorrência da infecção como evento adverso, resultante de um conjunto de aspectos. Ainda que se refiram imediatamente ao paciente, aos seus aspectos individuais, enquadra-se na perspectiva das condições de prestação da assistência à saúde, ou seja, o paciente apresenta-se em risco de adquirir IRAS pelo próprio contexto em que se encontra, em decorrência de uma prática profissional que não adote diretrizes de segurança para prevenção e controle de infecções ${ }^{(1,4)}$.

Na perspectiva dessa problemática, considera-se que a prevenção e o controle de IRAS são inerentes aos cuidados de enfermagem, medidas simples e efetivas podem prevenir e reduzir riscos e danos ${ }^{(5-6)}$. Para este empreendimento, a figura do enfermeiro-líder se destaca por encontrar-se na linha de frente do processo assistencial, sendo o sujeito que pode deflagrar o tipo e as estratégias de liderança adotadas para implementação de ações planejadas ${ }^{(7-8)}$. Contudo, este aspecto configura uma lacuna no conhecimento global, delineando-se como prioridade a elaboração de estratégias de liderança para a assistência segura ${ }^{(9)}$.

O pensamento complexo trouxe a base filosófica para sustentar este estudo ${ }^{(10)}$, pela demanda do enfermeiro-líder em encarar a diversidade de situações no cotidiano gerencial. A estratégia é entendida como ação, supõe a complexidade, é pensar em movimento, acaso, imprevisto, iniciativa, e das transformações ${ }^{(10)}$.

Diante da natureza complexa da problemática, apresentam-se como objeto de estudo estratégias de liderança em enfermagem para incorporação de medidas de prevenção e controle de IRAS em pacientes com câncer. O objetivo deste estudo foi elaborar estratégias de liderança em enfermagem voltadas à incorporação de medidas de prevenção e controle de IRAS em pacientes com câncer.

\section{METODOlOGiA}

Trata-se de estudo desenvolvido pelo método de Pesquisa Convergente Assistencial (PCA), cujo desenho buscou delinear o encontro que envolve questões da investigação científica e da prática assistencial ${ }^{(11)}$ em um hospital habilitado como Unidade de Assistência de Alta Complexidade em Oncologia (UNACON), situado no município de Juiz de Fora, em Minas Gerais.

Os participantes do estudo foram selecionados de acordo com os seguintes critérios de inclusão: ser enfermeiro-líder atuante na gerência do processo assistencial nos setores de internações e trabalhar na instituição há mais de três meses.Como critérios de exclusão, foram definidos: os profissionais que verbalizaram recusa em participar da pesquisa; os profissionais lotados na central de material esterilizado, no centro cirúrgico e na auditoria em enfermagem; ou que estiveram ausentes no período de coleta de dados por motivo de férias e licenças diversas.

O período de coleta dos dados ocorreu no ano de 2013. No momento da coleta de dados, 20 profissionais totalizavam o quadro de enfermeiros na instituição, sendo que 16 atenderam aos critérios de inclusão e, destes, um não aceitou participar do estudo.

Foi desenvolvido com os 15 enfermeiros que aceitaram participar da pesquisa, num processo de sensibilização e identificação de situações-problemas enfrentadas na implementação das medidas de 
prevenção e controle de IRAS junto aos profissionais de enfermagem. A análise desses dados das situações-problemas subsidiou a formulação de questões norteadoras para elaboração das estratégias de liderança por meio de um grupo de discussão. Os enfermeiros foram consultados para ajustar o melhor horário e local para realização do grupo de discussão e optaram pelo centro de estudos do próprio hospital. No dia programado, compareceram nove enfermeiros, posteriormente os demais justificaram sua ausência, que se relacionou aos imprevistos de ordem pessoal e profissional.

Adotou-se a noção de pequeno grupo para a produção dos dados,em que é considerado como um sistema vivo formado por indivíduos-sujeitos humanos, possuidores de linguagem, cultura e consciência e como atividade humana essencial no processo de produção e organização social ${ }^{(12)}$. Para sistematizar os aspectos operacionais, destacou-se o planejamento, o aquecimento e o trabalho teórico como as atividades referentes ao enquadre do trabalho grupal ${ }^{(13)}$.

Foram elaboradas questões iniciais que representavam situações-problemas vivenciadas por eles no processo de liderança para conduzir o trabalho no grupo, as quais foram lançadas na intenção de instigar a discussão e reflexão sobre as estratégias de liderança que poderiam adotar ou já adotavam para atuar naquela questão. $\mathrm{O}$ critério escolhido para encerramento da coleta de dados foi o de saturação das falas dos participantes, quando o grupo chegava ao acordo de que deveria passar para outro ponto de discussão ${ }^{(13)}$. O grupo permaneceu reunido durante 2 horas.

O tratamento dos dados foi realizado por meio do software Análise Lexical Contextual de um Conjunto de Segmentos de Texto (Alceste) ${ }^{(14)}$. Após transcrição das falas dos participantes, o texto foi preparado de acordo com as orientações do Alceste, formando o corpus de análise dos dados. O corpus analisado foi formado por uma única unidade de contexto inicial (UCI), ou seja, o corpus foi analisado num único discurso, pois interessava, na organização coletiva das estratégias, identificar no mundo lexical elaborado pelo grupo de enfermeiros-líderes quais as estratégias de liderança em enfermagem voltadas à incorporação de medidas de prevenção e controle de IRAS em pacientes com câncer, como se configuravam e as possibilidades e desafios para serem implementadas na prática.

A organização para análise e interpretação dos dados foi de acordo com a partição das cinco classes que emergiram como produto da análise dos dados desenvolvida pelo programa. No software Alceste, as classes representam os aspectos abordados pelos participantes ${ }^{(14)}$.

A pesquisa foi aprovado em abril de 2012 pelo Comitê de Ética em Pesquisa da Universidade Federal do Rio de Janeiro sob o protocolo $\mathrm{n}^{\circ} 14.714$.

\section{RESULTADOS}

O perfil dos nove enfermeiros que participaram do grupo de discussão mostra que sete eram do gênero feminino e dois do masculino. Em relação à faixa etária, três deles tinham entre 20 e 30 anos, seis entre 30 e 40 anos. No que tange ao tempo de atuação dos enfermeiros na instituição, cinco trabalhavam entre seis meses e um ano, três trabalhavam entre um e cinco anos, e um trabalhava entre cinco anos e dez anos.

A Figura 1 é produto da análise do Alceste, mostra o dendograma no qual é possível verificar que o corpus se dividiu em cinco classes. Cada classe recebeu uma denominação conforme suas unidades de contexto elementares (UCE) e dos vocábulos mais frequentes e significativos sendo demonstrados pelo maior qui-quadrado (khi2). A ordem adotada para descrição das classes está de acordo com a sequência apresentada na Figura 1.

\section{Classe 1 - A presença do enfermeiro-líder nos setores de internação}

A Classe 1 representa 17\% do material classificado para análise. Embora as palavras terapia, intensiva e unidade tenham o maior khi2, os enfermeiros consideraram que as enfermarias eram os locais onde existiam mais situações-problemas, como se verifica na UCE a seguir:

[...] no setor fechado você está ali, está vendo, existe um controle melhor, nas enfermarias temos que trabalhar mais a questão da confiança, você não está lá o tempo inteiro. (UCE 164) 


\begin{tabular}{|c|c|c|c|c|c|c|c|c|c|}
\hline \multicolumn{2}{|c|}{ Classe 1} & \multicolumn{2}{|c|}{ Classe 2} & \multicolumn{2}{|c|}{ Classe 3} & \multicolumn{2}{|c|}{ Classe 5} & \multicolumn{2}{|c|}{ Classe 4} \\
\hline Vocábulo & Khi2 & Vocábulo & Khi2 & Vocábulo & Khi2 & Vocábulo & Khi2 & Vocábulo & Khi2 \\
\hline Terapia & 62 & Dia & 17 & Mãos & 69 & Pensar & 22 & Infecção & 75 \\
\hline Intensiva & 62 & Setor & 17 & Lavagem & 68 & Forma & 18 & Hospitalar & 75 \\
\hline Unidade & 57 & Adaptou & 11 & Gel & 36 & Equipe & 14 & Serviço & 74 \\
\hline Enfermarias & 22 & Tempo & 10 & Álcool & 36 & Pode & 11 & Controle & 50 \\
\hline Vaga & 20 & Dar & 9 & Medicação & 17 & Seja & 8 & Papel & 40 \\
\hline Vendo & 7 & Vir & 9 & Conscientizar & 13 & Falha & 8 & Enfermeira & 31 \\
\hline Cuidado & 7 & Demorar & 6 & Cheia & 12 & Continuada & 8 & Antiga & 23 \\
\hline Falta & 6 & Qualidade & 6 & Paciente & 11 & Necessidade & 8 & Culturas & 12 \\
\hline Descer & 5 & Veem & 5 & Passar & 8 & Orienta & 7 & Importância & 9 \\
\hline Existe & 5 & Ganha & 4 & Troca & 6 & Acho & 6 & Ver & 4 \\
\hline Vê & 3 & Conhece & 4 & Posto & 6 & Mostrar & 6 & Atitude & 4 \\
\hline Grave & 3 & Educação & 4 & Melhor & 6 & Aspecto & 6 & Importante & 4 \\
\hline Convênio & 3 & Horário & 4 & Problemas & 6 & Atitude & 6 & Centro & 3 \\
\hline $\begin{array}{l}\text { Primeiro } \\
\text { andar }\end{array}$ & 3 & Andar & 3 & Física & 4 & Partir & 4 & Instituição & 3 \\
\hline $\begin{array}{l}\text { Terceiro } \\
\text { andar }\end{array}$ & 3 & Mês & 3 & Estrutura & 4 & Quer & 4 & Enfermagem & 3 \\
\hline
\end{tabular}

Figura 1 - Esquema estrutural temático das estratégias de liderança elaboradas. Rio de Janeiro, 2016

As palavras enfermarias, vaga, cuidado e grave revelam que nem sempre há vaga ou o paciente tinham indicação de internação na Unidade de Terapia Intensiva (UTI), como é possível verificar na UCE a seguir:

Eu já tive momentos no terceiro [refere-se a um setor de internação] que tinha sete pacientes fora de possibilidade terapêutica, que precisavam de cuidados de unidade de terapia intensiva, não desceu porque não tinha possibilidade para descer para não ocupar a vaga. (UCE 36)

\section{Classe 2 - O processo de trabalho do enfermeiro-líder}

A Classe 2 compõe 37\% do discurso analisado, é a maior classe e apresenta como palavras mais expressivas: dia, setor, adaptação e tempo, conforme pode ser identificado na UCE a seguir:

Quando você trabalha um bom tempo naquele setor, você ganha tempo, você conhece os clientes, você acompanha todo o dia [...]. (UCE 153)

[...] ajudá-lo a se encontrar. Já tivemos casos de colegas, de técnicos, que passaram por mim, passaram pelo convênio, foi para outro setor, voltou para mim agora e se adaptou [...]. (UCE 155)

As palavras educação e horário destacam os desafios para os profissionais comparecerem às atividades educativas. Assim o enfermeiro deve assumir uma postura integradora da educação permanente com o dia a dia dos profissionais, como aponta a UCE a seguir:

O multiplicador é o enfermeiro, é o supervisor enfermeiro, ele tem que ter ciência da palestra e ficar atento para multiplicar. Eu vejo assim, se ele tem essa dificuldade que eu estou colocando em relação ao horário para liberar alguém [...]. (UCE 95)

\section{Classe 3 - A higienização das mãos na prevenção e controle de infecções}

A Classe 3 é composta por 15\% do discurso analisado, nela se sobressai a noção de elementos que envolvem a Higiene das Mãos (HM). A afirmação da pragmática da HM se respalda em palavras com maior significância, como mãos, lavagem, gel e álcool, conscientização, como mostra a UCE a seguir:

E não se cria o hábito de lavar as mãos, cria-se o hábito de não lavar, passa pela questão da conscientização. 
Eu acho que é muita falta de preparo do pessoal, o câncer em si propicia muita falta de imunidade, e, em geral, não só os técnicos, os enfermeiros, estão tendo um preparo que não é mais o mesmo. (UCE 16)

Outro elemento que o discurso dos enfermeiros aponta foi a necessidade de envolver os pacientes e acompanhantes para desenvolver o hábito de HM, conforme é visto no trecho ilustrativo da UCE:

[...] aí acontecem os erros, as infecções e tudo mais não são percebidos, é porque as pessoas também não ajudam os funcionários. Mas isso nem sempre é bem recebido, paciente que cobra isso demais o técnico avisa, aquele é chato, um passa para o outro, olha aquele cobra o uso do álcool gel antes de tocá-Io. (UCE 128)

As palavras problemas, estrutura e física trazem a informação de que as ações desencadeadas para HM sofrem influências do tipo de infraestrutura da instituição, conforme pode ser visto na UCE a seguir:

São problemas que temos que passar para a direção, fazer um levantamento de dados, como os problemas que estão acontecendo, quais seriam as soluções, melhoria da estrutura física caberia ter esse levantamento. (UCE 76)

\section{Classe 4 - A enfermeira da Comissão de Controle de Infecção Hospitalar, parceira do enfermeiro-líder}

A Classe 4 obteve uma representatividade de $12 \%$ do material analisado e se expressa pelas palavras infecção, hospitalar, serviço, controle, papel e enfermeira. Estas são ilustrativas da tendência dessa classe que evidencia as competências do enfermeiro da Comissão de Controle de Infecção Hospitalar $(\mathrm{CCIH})$, como se verifica na UCE a seguir:

A gente também tem que supervisionar, também tem que olhar. Estamos mais conscientes que temos que trabalhar juntos, nós e a enfermeira do serviço de controle de infecção hospitalar, ela tem que estar inserida na nossa rotina de trabalho. (UCE 171)

\section{Classe 5 - A promoção do conhecimento de IRAS para a equipe de enfermagem}

A Classe 5 é composta por $19 \%$ do discurso analisado, nela sobressaem os elementos que envolvem a forma de pensar da equipe sobre a prevenção e o controle de IRAS nos pacientes com câncer. Nessa classe, as palavras mais significativas foram: pensar, forma, equipe e pode, que reforçam a noção de que o discurso dos enfermeiros se encaminha para pensar a prevenção e o controle de infecções de uma forma coletiva. Tal afirmação é exemplificada pela seguinte UCE:

Pensar que pode ser de uma forma coletiva a toda a equipe, só para ressaltar, é o ensino do dia a dia, viu a falha, vai e corrige, orienta, só para ressaltar, todo mundo tem que falar a mesma língua, não adianta um só falar e o outro não. (UCE 5)

A presença das palavras continuada, necessidade e orientação reforça a inferência acerca da necessidade de buscar estratégias para que a formação seja continuada e permanente nesta instituição, como aponta a UCE a seguir:

Essa questão de divulgar realmente essa necessidade de orientação, um modo para prover essa educação continuada para toda a equipe e demonstrar sempre que o paciente, aqui no caso o oncológico, ele não vem aqui de uma forma para morrer, sim para ter uma perspectiva de vida, ele quer ser curado. (UCE 51)

As palavras orienta e acho, quando visualizadas nas UCEs, estavam ligadas à orientação que os sensibiliza sobre o tema, conforme pode ser conferido na UCE a seguir:

Eu acho que essas coisas assim passam por uma educação, por uma cobrança, mudança de atitude é muito complicado, passa pela conscientização de cada um, tem coisas que a gente orienta, orienta, orienta e eles não se conscientizam (UCE 9). 
Os participantes do estudo evidenciam que as unidades tipo enfermarias são os setores com mais situações-problemas, devido ao fato de os pacientes com câncer requerem elevadas horas de atendimento de enfermagem, independentemente do setor em que estão internados, e por outro lado, existe um quantitativo de profissionais aquém da demanda dos cuidados nesses setores.

As estratégias de liderança devem ser implementadas em todos os setores do hospital, mas os locais prioritários para começar esse empreendimento são as enfermarias. Esse entendimento relaciona-se a uma característica do trabalho dos enfermeiros na instituição, de assumirem outros setores concomitantemente, e com isso não conseguirem acompanhar o trabalho da equipe como gostariam. Situação que aponta para uma reflexão sobre a relação do dimensionamento de pessoal com a demanda de cuidados de enfermagem para os pacientes com câncer. Tal visão é condizente com a concepção atual do dimensionamento de pessoal de enfermagem, entendida por previsão do quantitativo de pessoal como um processo que depende do conhecimento da carga de trabalho existente nas unidades de internação e do trabalho, relaciona-se com as necessidades de assistência dos pacientes e do padrão de cuidado pretendido ${ }^{(15)}$.

Além disso, os resultados apontam a tendência de promover estratégias para fortalecer a adaptação dos profissionais aos setores de internações. A questão da adaptação é relacionada com a rotatividade no trabalho e experiência do profissional na oncologia ${ }^{(16)}$, sendo vista concomitantemente como facilitadora e dificultadora. Isso porque compete ao enfermeiro saber lidar e tirar proveito para melhorar o processo de trabalho, por exemplo, a experiência pode conferir um excesso de confiança, bem como a inexperiência pode ocasionar a inobservância da incorporação das medidas de prevenção e controle de IRAS.

Os participantes apontam que o enfermeiro-líder precisa promover condições para obter a participação dos profissionais nas propostas educativas. A educação permanente é considerada uma estratégia que busca promover aprendizagem dos profissionais, sendo indicada para adaptá-los ao processo de trabalho. Na lógica da educação permanente, as ações de educação transpassam a atualização de conhecimentos, buscando ensinar a problematizar, questionar, repensar e reinventar o cotidiano dos serviços de saúde ${ }^{(17)}$.

Para eficácia de estratégias educativas sobre prevenção e controle de IRAS, destaca-se a importância de discutir questões que envolvem a participação dos profissionais nas aulas, como o tempo, a demora em acontecer e o melhor horário para atraí-los ${ }^{(18)}$. Na prática assistencial é preciso estimular os profissionais a participar dessas programações e aprender a apreender o conteúdo abordado para imprimi-lo em sua prática de cuidados ${ }^{(19)}$.

O grupo de enfermeiros leva o discurso para uma linha de argumentação em que é destacada a competência do enfermeiro como integradora da educação permanente com o dia a dia dos profissionais. Quando é observado algum erro, cujo destaque é dado pelos participantes à inobservância da $\mathrm{HM}$, deve ser entendido numa linha de pensamento que busque a sensibilização dos profissionais para o risco dos pacientes com câncer em desenvolver infecções, acentuado pela baixa imunidade.

Pensar no gerenciamento de riscos para IRAS, desafio posto pelos enfermeiros para trabalhar com o erro, aponta para estratégias de intervenção que sejam iniciadas no ato de identificação da falha ${ }^{(4)}$. O erro é um problema primário, original, prioritário, sobre o qual ainda há muito que se pensar ${ }^{(10)}$. Projetar essa ideia ao comportamento dos profissionais é considerar que o erro é inerente às ações humanas, embora as diretrizes de segurança ressaltem que é no ato do fazer que o erro se materializa ${ }^{(1)}$.

É necessária uma visão capaz de articular a existência de multifatores que se integram para entender origem do erro e gerenciar os seus fatores de riscos, não focar somente em cobrança, mas centrar-se na construção do conhecimento num processo educativo contínuo, que visa incentivar e orientar os profissionais na execução de suas atividades.

A inobservância da HM é entendida como um problema que requer estratégias individuais e coletivas para melhorar a sua incorporação. Os fatores que influenciam na baixa adesão à HM são múltiplos, são aspectos que envolvem o profissional, assim como os seus conhecimentos, o valor atribuído, hábitos 
e crenças, além dos aspectos institucionais ligados à sobrecarga de trabalho e aos aspectos gerenciais da assistência ${ }^{(6)}$.

Os participantes destacaram que, para os profissionais realizarem a HM sempre que indicado, é preciso criar o hábito. Essa perspectiva é condizente com estudo que destaca a importância do hábito e da crença pessoal, que inclusive podem exercer maior influência na adesão do que o conhecimento das medidas de precaução e controle de infecção ${ }^{(20)}$.

Os problemas relacionados com a infraestrutura se configuram como desafios para a $\mathrm{HM}$, sendo recorrentes na instituição investigada. Higienizar as mãos também é um desafio em outros serviços de assistência à saúde em todo o mundo, é uma atitude dos profissionais influenciada pelas condições do trabalho, pelo modo como é organizado e pela infraestrutura física dos serviços de saúde ${ }^{(6,19)}$.

Outra estratégia capaz de favorecer a incorporação das medidas de prevenção e controle de infecções é a participação ativa dos pacientes e acompanhantes no processo assistencial. Salienta-se que essa estratégia necessita ser trabalhada a partir da democratização das relações de trabalho e da valorização dos profissionais da saúde, situação que requer uma ampla visão e está relacionada com as diretrizes da Segurança do Paciente numa relação de transversalidade com os profissionais, cuja atitude de HM é um dos requisitos da prestação de um atendimento seguro(1).

A estratégia embasa-se em orientar os pacientes e lhes dar a oportunidade de se interessar pelo seu cuidado. Contudo, os enfermeiros enfatizam que também é preciso trabalhar esse aspecto com a equipe de enfermagem, pois ainda se tem uma cultura na qual os profissionais de saúde não estão habituados a ser questionados pelos pacientes durante a prestação da assistência.

O grupo de enfermeiros possui uma visão que coloca a IRAS como uma condição também resultante da forma como a assistência é prestada. Assim a orientação fornecida para os profissionais se configura como ação capaz de influenciá-los a desenvolver determinada atitude ${ }^{(18)}$. O discurso dos enfermeiros remete à ideia de que o profissional pode ser ativo nas ações de prevenção e controle de IRAS, quando sua forma de pensar o conduz para uma visão capaz de gerenciar os riscos, distanciando a infecção do aspecto de ser uma fatalidade na oncologia.

É possível perceber que os enfermeiros relacionam esse aspecto distante da realidade deles, na qual ainda é considerado incipiente o conhecimento referente à prevenção e ao controle de IRAS, tanto na formação profissional quanto em serviço. Para que a transformação da realidade possa ser concretizada, há necessidade de estratégias que empreendam esforços direcionados para a formação profissional, com foco no conhecimento, consciência crítica e posicionamento ético ${ }^{(10)}$.

Os participantes referem à mudança de perspectiva do enfermeiro da $\mathrm{CCIH}$, que, no passado, era visto como profissional distante dos demais que estavam na linha de frente da assistência e atualmente é reconhecido como um parceiro, pois tem um olhar voltado às ações específicas de prevenção e controle de infecções. Dado condizente com estudo em que os enfermeiros associam o desempenho da $\mathrm{CCIH}$ à figura do enfermeiro da $\mathrm{CCIH}$, consideram-no como agente fundamental para implementação das ações do Programa de Controle de Infecção Hospitalar ${ }^{(21)}$.

Os resultados evidenciam a importância da interação entre os profissionais como uma estratégia para promover a segurança dos pacientes, pela integração e otimização dos processos de trabalho, que, na prática, se apresentam inter-relacionados. A relação entre os profissionais é um processo que precisa da integração deles no planejamento e na implementação das ações, caso contrário, as medidas de prevenção e controle de IRAS não se sustentam na prática.

\section{CONSIDERAÇÕES FINAIS}

Elaborar estratégias de liderança de forma coletiva com enfermeiros que atuam na oncologia constitui instigante perspectiva, tendo em vista que o objetivo das discussões foi desmitificar a ocorrência de infecção em pacientes com câncer como um evento esperado, e colocar num patamar cujo risco pode ser gerenciado. Assim, as estratégias de liderança são apostas empreendidas de acordo com a situação, para dar conta da imprevisibilidade presente no contexto e da necessidade de adotar uma atitude educativa, para favorecer a mudança de comportamento dos profissionais de enfermagem. 
Enfatiza-se que as ações saem da perspectiva de atuação exclusiva junto ao profissional para construir uma relação de transversalidade, quando buscam incluir os pacientes e acompanhantes sobre o direito de posicionarem-se frente aos profissionais de saúde. Nesse modo de pensar integrador e participativo, o desafio posto pelos enfermeiros no gerenciamento de riscos para IRAS é trabalhar com o erro na perspectiva de articular a existência de multifatores que se integram para sua ocorrência.

As estratégias foram elaboradas adotando-se um modo de pensar integrador e participativo, num movimento de reflexão-ação-reflexão e contemplou-se a dinamicidade do contexto de atuação e a dialogicidade entre os sujeitos envolvidos no processo de liderança. Assim, a construção coletiva das estratégias de liderança impactou a redução da dicotomia entre o fazer e o pensar, o que pode contribuir para convergência da ciência de enfermagem com a prática de enfermagem.

Desenvolver um estudo na perspectiva da PCA se configurou como um desafio para atender a premissa de uma pesquisa coparticipativa, pois, na prática assistencial, os enfermeiros continuam em suas atividades, e dispor de um tempo para participarem do grupo visando promover estratégias coletivas não foi tarefa fácil. Essa questão culminou como uma limitação do estudo, cujo contexto implicou no processo de pesquisa ao não favorecer a participação dos enfermeiros. Assim, futuros estudos que contemplem estratégias metodológicas para construções coletivas de estratégias de liderança poderão contribuir para o avanço do conhecimento da enfermagem.

\section{REFERÊNCIAS}

1. Ministério da Saúde (BR). Agência Nacional de Vigilância Sanitária (ANVISA). Medidas de Prevenção de Infecção Relacionada à Assistência à Saúde. Série Segurança do Paciente e Qualidade em Serviços de Saúde. Brasília: Ministério da Saúde/ ANVISA; 2013.

2. Kamboj M, Sepkowitz KA. Nosocomial infections in patients with cancer. Lancet Oncol.[Internet] 2009; 10(6) [acesso em 05 out 2013]. Disponível: http://dx.doi.org/10.1016/S1470-2045(09)70069-5.

3. Santos SLV, Sousa BTK, Costa DM, Lopes LKO, Pelajad EB, Sousa DM, et al. Infecções associadas ao cuidado em saúde em um hospital oncológico brasileiro: análise de cinco anos. Enferm. glob. [Internet] 2012; (25) [acesso em 30 maio 2015]. Disponível: http://scielo.isciii.es/pdf/eg/v11n25/pt_clinica2.pdf.

4. Feldman BL. Gestão em Enfermagem: ferramenta para a prática segura. In: Cunha ICKO, Harada MJCS, organizadores. Gestão de risco: implicações para a prática. São Caetano do Sul (SP): Yendis, 2011.

5. Giarola LB, Baratieri T, Costa AM, Bedendo J, Marcon SS, Waidman MAP. Infecção hospitalar na perspectiva dos profissionais de enfermagem: um estudo bibliográfico. Cogitare Enferm. [Internet] 2012; 17(1) [acesso em 30 maio 2015]. Disponível: http://dx.doi.org/10.5380/ce.v17i1.26390.

6. Huis A, Holleman G, van Achterberg T, Grol R, Schoonhoven L, Hulscher M. Explaining the effects of two different strategies for promoting hand hygiene in hospital nurses: a process evaluation alongside a cluster randomised controlled trial. Implementation Sci. [Internet] 2013; (8) [acesso em 30 maio 2015]. Disponível: http:// dx.doi.org/10.1186/1748-5908-8-41.

7. Lanzoni GMM, Meirelles BHS. Leadership of the nurse: an integrative literature review. Rev. Latino-Am. Enfermagem. [Internet] 2011; 19(3) [acesso em 30 maio 2015]. Disponível: http://dx.doi.org/10.1590/S010411692011000300026.

8. Balsanelli AP, Cunha ICKO. Liderança ideal e real dos enfermeiros de unidade de terapia intensiva em hospitais privados e públicos. Cogitare Enferm. [Internet] 2016; 21(1) [acesso em 10 fev 2016]. Disponível:http://dx.doi. org/10.5380/ce.v21i4.42129.

9. Manning ML. Expanding infection preventionists' influence in the 21st Century: Looking back to move forward. Am J Infect Control. [Internet] 2010; 38(10) [acesso em 15 dez 2015]. Disponível: http://dx.doi.org/10.1016/j. ajic.2010.08.006.

10. Morin E. Ciência com consciência. 14ª ed. Rio de janeiro: Bertrand; 2010.

11. Paim L, Trentini M, Madureira VSF, Stamm M. Pesquisa convergente-assistencial e sua aplicação em cenários 
da enfermagem. Cogitare Enferm. [Internet] 2008; 13(3) [acesso em 15 dez 2015]. Disponível: http://dx.doi. org/10.5380/ce.v13i3.12990.

12. Alves MC, Seminotti N. O pequeno Grupo e o paradigma da Complexidade de Edgar Morin. Psicol. USP. [Internet] 2006; 17(2) [acesso em 15 dez 2013]. Disponível: http://dx.doi.org/10.1590/S0103-65642006000200006.

13. Mota KAMB, Munari DB. Um olhar para a dinâmica do coordenador de grupos. Rev. Eletr. Enf. [Internet] 2006; 8(1) [acesso 05 set 2013]. Disponível: http://www.fen.ufg.br/revista/revista8_1/atualizacao.htm.

14. Lima LC. Programa Alceste, primeira lição: a perspectiva pragmatista e o método estatístico. Rev Edu. Pública. 2008; 17(33): 83-97.

15. Maya CM, Simões ALA. Implicações do dimensionamento do pessoal de enfermagem no desempenho das competências do profissional enfermeiro. Rev.bras.enferm. [Internet] 2011; 64(5) [acesso em 05 set 2015]. Disponível: http://dx.doi.org/10.1590/S0034-71672011000500015.

16. Bordignon M, Monteiro MI, Mai S, Martins MFSV, Rech CRA, Trindade LL. Satisfação e insatisfação no trabalho de profissionais de enfermagem da oncologia do Brasil e Portugal. Texto Contexto Enferm. [Internet] 2015; 24(4) [acesso em 15 jan 2016]. Disponível: http://dx.doi.org/10.1590/0104-0707201500004650014.

17. Sade PMC, Peres AM, Pedroso JVM, Larocca LM. Núcleo de enfermeiros de Educação Permanente do Paraná: trajetória e contribuições. Cogitare Enferm. [Internet] 2016; 21(3) [acesso em 02 jul 2016]. Disponível: http://dx.doi. org/10.5380/ce.v21i2.44335.

18. Gonçalves FAF, Brasil VV, Minamisava R, Caixeta CR, Oliveira LMAC, Cordeiro JABL. Eficácia de estratégias educativas para ações preventivas da pneumonia associada à ventilação mecânica. Esc. Anna Nery. [Internet] 2012; 16(4) [acesso em 15 jan 2016]. Disponível: http://dx.doi.org/10.1590/S1414-81452012000400023.

19. Bathke J, Cunico PA, Maziero ECS, Cauduro FLF, Sarquis LMM, Cruz EDA. Infraestrutura e adesão à higienização das mãos: desafios à segurança do paciente. Rev. Gaúcha Enferm. [Internet] 2013; 34(2) [acesso em 15 jan 2016]. Disponível: http://dx.doi.org/10.1590/S1983-14472013000200010.

20. Oliveira AC, Cardoso CS, Mascarenhas D. Contact precautions in intensive care units: facilitating and inhibiting factors for professionals'adherence. Rev. esc. enferm. USP. [Internet] 2010; 44(1) [acesso em 15 jan 2016]. Disponível: http://dx.doi.org/10.1590/S0080-62342010000100023.

21. Delage DGA, da Silva GA. Prevenção e controle das infecções hospitalares: um desafio em instituições de saúde de Juiz de Fora. Rev. Baiana Saúde Pública. [Internet] 2011; 35(4) [acesso em 15 jan 2016]. Disponível: http:// inseer.ibict.br/rbsp/index.php/rbsp/article/view/267. 